\title{
Molecular Cloning and Characterization of the First Caspase in the Striped Stem Borer, Chilo suppressalis
}

\author{
Ming-Xing Lu ${ }^{1,2}$, Yu-Zhou Du ${ }^{1, *}$, Shuang-Shuang Cao ${ }^{1}$, Pingyang Liu ${ }^{2}$ and Jianyong $\mathrm{Li}^{2, *}$ \\ 1 College of Horticulture and Plant Protection \& Institute of Applied Entomology, \\ Yangzhou University, Yangzhou 225009, Jiangsu, China; \\ E-Mails: lumingxing0123@yahoo.com.cn (M.-X.L.); caosshuang@163.com (S.-S.C.) \\ 2 Department of Biochemistry, Virginia Tech, Blacksburg, VA 24061, USA; \\ E-Mail: lpingyang1987@gmail.com \\ * Authors to whom correspondence should be addressed; E-Mails: yzdu@yzu.edu.cn (Y.-Z.D.); \\ lij@vt.edu (J.L.); Tel./Fax: +86-514-8797-1854 (Y.-Z.D.); Tel.: +1-540-231-1182 (J.L.); \\ Fax: +1-540-231-9070 (J.L.).
}

Received: 8 April 2013; in revised form: 17 April 2013 / Accepted: 3 May 2013 /

Published: 15 May 2013

\begin{abstract}
Apoptosis is executed through the activity of the caspases that are aspartyl-specific proteases. In this study, we isolated the caspase gene (Cscaspase-1) of Chilo suppressalis (one of the leading pests responsible for destruction of rice crops). It possesses the open reading frame (ORF) of 295 amino acids including prodomain, large subunit and small subunits, and two cleavage sites ( $\mathrm{Asp}^{23}$ and $\mathrm{Asp}^{194}$ ) were found to be located among them. In addition to these profiles, Cscaspase-1 contains two active sites $\left(\mathrm{His}^{134}\right.$ and $\mathrm{Cys}^{176}$ ). Genomic analysis demonstrated there was no intron in the genome of Cscaspase-1. The Cscaspase-1 transcripts were found in all tissues of the fifth instar larvae, and higher levels were found in the midgut, hindgut and Malpighian tubules. Examination of Cscaspase-1 expression in different developmental stages indicated low constitutive levels in the eggs and early larvae stages, and higher abundances were exhibited in the last larvae and pupae stages. The relative mRNA levels of Cscaspase-1 were induced by heat and cold temperatures. For example, the highest increase of Cscaspase- 1 transcription was at $-3{ }^{\circ} \mathrm{C}$ and $36{ }^{\circ} \mathrm{C}$ respectively. In a word, Cscaspase-1 plays a role of effector in the apoptosis of $C$. suppressalis. It also correlates with development, metamorphosis and thermotolerance of $C$. suppreassalis.
\end{abstract}


Keywords: apoptosis; Cscaspase-1; Chilo suppressalis; expression; development; thermotolerance

\section{Introduction}

Although apoptosis was first described in the publication over forty years ago [1], it still draws great attention as a result of its role in development, host defense, and a response of cells to general stress [2-5]. Apoptosis is executed through the activity of the caspases that are aspartyl-specific proteases [6,7]. The caspase family is divided into two groups: the initiators and the executors of apoptosis, each including three domains: an amino terminal domain, a large subunit and a small subunit [8]. In insects, caspases also play an important role in apoptosis [9]. The Drosophila genome contains seven caspase genes that function in both immune signaling pathways and development. In Aedes aegypti, Aedes Dronc, a kind of caspase gene, also possesses similar roles [10,11]. Five caspase family members were found in Bombyx mori genome [12]. 66 caspase sequences representing 27 lepidopteran insect species can be classified into at least five groups by phylogenetic analyses [13]. However, completed studies on the genome, structure and functions of caspases in insect were found infrequently, especially the relationship with thermotolerance [5,14-17].

The thermotolerant ability of insect can be divided into basal and inducible components [18]. When exposed to adverse temperatures, organisms may respond differently including the induction of caspases that causes the apoptosis. For example, low temperature led to apoptosis in some mammalian cells [19], and high temperature could significantly promote apoptosis process resulting in the increased mortality rate of Plutella xylostella [16]. The caspase is also related to the cold hardness in Drosophila melanogaster [5]. In order to understand the molecular mechanism of thermotolerance of insect, it's necessary to study the relation between caspase and thermotolerance.

The striped stem borer, Chilo suppressalis (Walker) (Insecta: Lepidoptera: Pyralidae), is one of the most insect pests of rice in Asia, Northern Africa and Southern Europe, and has a complex life cycle. According to our studies in 2010, field-collected larvae in March could survive at $-21^{\circ} \mathrm{C}$ [20], and the field larvae in summer could tolerance $46{ }^{\circ} \mathrm{C}$ in Yangzhou area, Jiangsu province, China. In the present work, we set out to investigate and isolate caspase-1 in C. suppressalis. Hereafter, its structure model was predicted. At the same time, caspase-1 $m$ RNA expression of different tissues (organs), developmental stages and temperatures was demonstrated.

\section{Results and Discussion}

\subsection{Isolation, Cloning, Sequencing and Structure of Cscaspase-1}

Degenerate primers based on conserved regions from several insect caspase- 1 were used to amplify a 359 bp partial fragment from $C$. suppressalis cDNA. The cloned fragment was sequenced and a BLAST analysis of its deduced amino acid fragment revealed apparent sequence homology with the caspase-1 (data not shown). The full-length 1422-bp Cscaspase-1, including the UTRs, was obtained through 5' and 3' RACE (GenBank accession no. JQ864206). It possesses the open reading frame 
(ORF) of 295 amino acids with a molecular weight of $33.56 \mathrm{kDa}$ and an isoelectric point of 6.2. Two cleavage sites were found to be located between the prodomain and large subunit at $\mathrm{Asp}^{23}$, and between the large and small subunits at Asp ${ }^{194}$. Moreover, Cscaspase-1 contains QACQG pentapeptide active-site motif, which is also found in lepidopteran caspase-1 (Figure 1A) [5,14-17].

Figure 1. The sequence and structure analysis of the Cscaspase-1. (A) Nucleotide and deduced amino acid sequence of Cscaspase-1 (JQ864206). Two cleaved sites to generate the large and small subunit are also shown by red dots. The active site pentapeptide QACQG was boxed; (B) Homology modeling of the Cscaspase-1 (light yellow) with Spodoptera frugiperda caspase-1 (PDB ID: 2NN3) (cornflower blue) as template using Phyre2 software; (C) The large (p19) and small subunit (p12) regions respectively are shown in blue and red. TETDG motifs are shown as red spheres and two active sites (His ${ }^{134}$ and $\mathrm{Cys}^{176}$ ) are shown as red sticks.

A ACATGGGGATGTCATGCATGTCATTTTCGGTTGTTGTGTAAATTGTAAATCCGTAATCGC 60 ATGGTGTTTTATTTTGAATAGTGTTTTACAAGGTTAAATAAAAATAGGGAATTGTTTCAA 120 TTACTAGGAAATCTGTGAATAGTAAAATAGGAGTTTTTTTTGTTTACAACTGATAATAAC 180 TTATCTTTCCGAAATGTCTGACGAAGAACTTAAAAGAAACAATGGTGCTGAACAAGAACA 240 $\begin{array}{lllllllllllllllll}M & S & D & E & E & L & K & R & N & N & G & A & E & Q & E & Q & 16\end{array}$ AAGGGAAAACGGCGGAGGCGACGAGGGCGACGCATGGGGTGGCCAAGGGTCTTCGGATAA 300

$\begin{array}{lllllllllllllllllllllll}R & E & N & G & G & G & D & E & G & D & A & W & G & G & Q & G & S & S & D & K & 36\end{array}$ AAATCGCTATGCCAAGATGCCTGTTGATAGAAATGCGCCATATTACAACATGAACCACAA 360 $\begin{array}{llllllllllllllllllllllll}N & R & Y & A & K & M & P & \text { V } & \text { D } & R & \text { N } & \text { A } & \text { P } & \text { Y } & \text { Y } & \text { N } & \text { M } & \text { N } & \text { H } & \text { K } & 56\end{array}$ GAATCGCGGTATGGCGTTGATTTTTAACCACGAACATTTTGATATCCATAGCTTGAAATC 420

$\begin{array}{llllllllllllllllllllll}\mathbf{N} & \mathrm{R} & \mathrm{G} & \mathrm{M} & \mathrm{A} & \mathrm{L} & \mathrm{I} & \mathrm{F} & \mathrm{N} & \mathrm{H} & \mathrm{E} & \mathrm{H} & \mathrm{F} & \mathrm{D} & \mathrm{I} & \mathrm{H} & \mathrm{S} & \mathrm{L} & \mathrm{K} & \mathrm{S} & 76\end{array}$ TCGCACAGGCACTAATGTCGACAGTGACAATCTGTCTAAGGTTCTGAGAGGCTTGGGATT 480

$\begin{array}{lllllllllllllllllllllll}R & T & G & T & N & V & D & S & D & N & \text { L } & S & K & V & \text { L } & R & G & \text { L } & G & F & 96\end{array}$ CCGAGTTACTGTTTTCCATAATCTTAAATCTGAAGAAATAAACAAATACATTCAGCAGAC 540

$\begin{array}{lllllllllllllllllllllll}R & V & T & V & F & H & N & L & K & S & E & E & I & N & K & Y & I & Q & Q & T & 116\end{array}$ AGCAGATATGGATCACTCAGATTCTGATTGTCTGTTGATTGCTGTGCTTACTCATGGTGA 600

$\begin{array}{lllllllllllllllllllll}\text { A } & D & \text { M } & \text { D } & \text { H } & \text { S } & \text { D } & \text { S } & \text { D } & \text { C } & \text { L } & \text { L } & \text { I } & \text { A } & \text { V } & \text { I } & \text { T } & \text { H } & \text { G } & \text { E } & 136\end{array}$ AATGGGTATGTTGTATGCTAAGGATACACATTACAAACCAGATAATTTGTGGTATTACTT 660 $\begin{array}{llllllllllllllllllllllll}M & G & M & \text { L } & Y & \text { A } & \text { K } & \text { D } & \text { T } & \text { H } & \text { Y } & \text { K } & \text { P } & \text { D } & \text { N } & \text { L } & \text { W } & \text { Y } & \text { Y } & \text { F } & 156\end{array}$ TACTGCTGACAAGTGCCCTTCCTTAGCAGGCAAACCTAAAATGTTTTTCATTCAAGCTTG 720

$\begin{array}{llllllllllllllllllllllllll}T & A & D & K & C & P & S & L & A & G & K & P & K & M & F & F & I & A & C & 176\end{array}$ CCAAGGTGATAAATTGGATGGAGGAATTACACTGATCAATCGTACTGAAACTGATGGTTC 780 \begin{tabular}{llllllllllllllllllllll}
\hline $\mathrm{Q}$ & $\mathrm{G}$ & $\mathrm{D}$ & $\mathrm{K}$ & $\mathrm{L}$ & $\mathrm{D}$ & $\mathrm{G}$ & $\mathrm{G}$ & $\mathrm{I}$ & $\mathrm{T}$ & $\mathrm{L}$ & $\mathrm{I}$ & $\mathrm{N}$ & $\mathrm{R}$ & $\mathrm{T}$ & $\mathrm{E}$ & $\mathrm{T}$ & $\mathrm{D}$ & $\mathrm{G}$ & $\mathrm{S}$ & 196
\end{tabular} TTCTAGCACCCAATACAGGATTCCTATTCATGCTGATTTCTTGATAGTGTTCTCCACTGT 840 $\begin{array}{lllllllllllllllllllllll}S & S & T & Q & Y & R & I & P & I & H & A & D & F & \text { L } & \text { I } & \text { V } & F & S & T & V & 216\end{array}$ GCCAGGATACTTTTCTTGGAGAAATACTACTCGTGGTTCTTGGTTTATGCAAGCTCTTTG 900

$\begin{array}{lllllllllllllllllllll}P & G & Y & F & S & W & R & N & T & T & R & G & S & W & F & M & Q & A & \text { L } & C & 236\end{array}$ TGATGAATTACGCTACTCAGGCACTGACAGAGACATACTCACTCTTCTGACCTTTGTTTG 960

$\begin{array}{lllllllllllllllllllll}D & E & I & R & Y & S & G & T & D & R & D & I & I & T & I & I & T & F & V & C & 256\end{array}$ TCAGAAAGTAGCATTAGATTATGAGTCCAACACTCCAGATACACTAACTATGCATCAGCA 1020

$\begin{array}{llllllllllllllllllllllllllllll}Q & K & V & A & I & D & Y & E & S & N & T & P & D & T & L & T & M & H & Q & Q & 276\end{array}$ GAAGCAAGTTCCTTGTCTTACCAGTATGCTCACCCGTTTATTTGTTTTTGGAAGAAAATA 1080

$\begin{array}{lllllllllllllllllllll}K & Q & V & P & C & L & T & S & M & L & T & R & L & F & V & F & G & R & K & * & 295\end{array}$ ATTTAATTTTACAAAATAATAATGTCCTTTGGTTTACCTACTGCTAGGTATAGTTTTT 1140 AATGGCTACTTGGTAAATGAGTTTGCCCTAGTTTTATTGATACAAAATGTTTATTGATAG 1200 AGATTTTTTTAATACATTGAGTACATACAAATTATTATTTTTTGTCACAATGCACTTTGA 1260 GATTATGATTAAGGTTATGTTTGATGAACTGGCTTCATCAATTCAATATATAGAGCAAT 1320 TATCATTTGTATCACCATGACTATTCATATAAATATTGTTTTAAACAAAATAGTTTATTC 1380 AGGAAACCTCACTCACAAAAAAAAAAAAAAAAAAAAAAAAAA 
Figure 1. Cont.

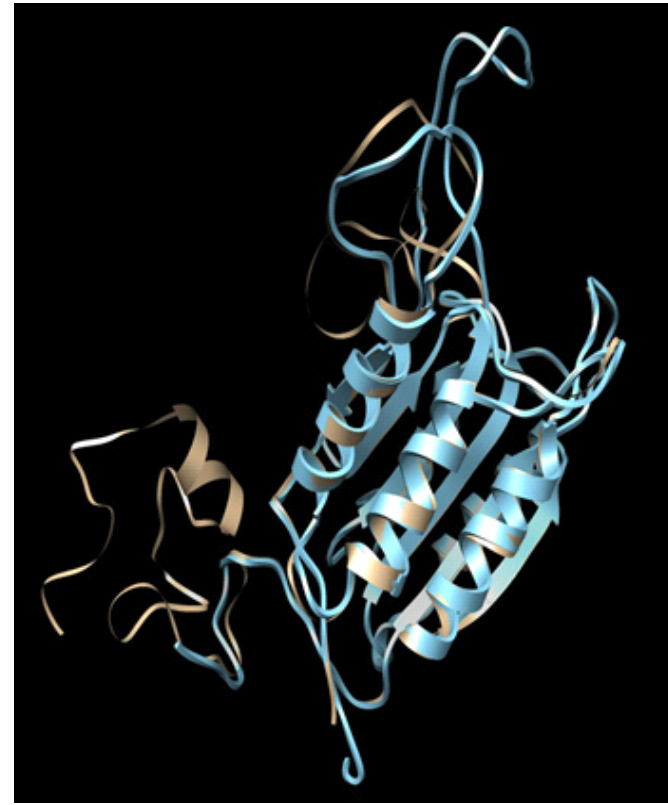

B

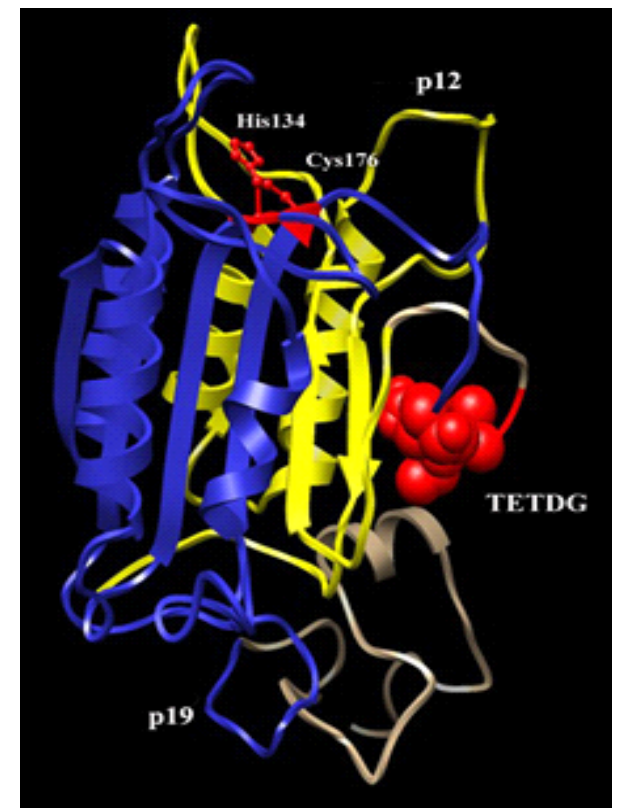

C

To investigate the potential structure-function relationship of Cscaspase-1, we generated its homology model with Phyre using Spodoptera frugiperda caspase-1 (PDB ID: 2NN3) as a template [14]. The structure of the Cscaspase-1 model was very similar to that of caspase-1 of $S$. frugiperda (confidence, 100\% statistically and identity, $87 \%$ based on sequence alignment) (Figure 1B). The model shows that Cscaspase-1contains three predicted domains, a prodomain, a large subunit domain (p19), and a small subunit domain (p12). Two active sites were found to be located at His ${ }^{134}$ and $\mathrm{Cys}^{176}$ respectively, which is known to be involved in executing apoptosis (Figure 1A,C). Through cleavage sites, pro-Cscaspase-1 would be cleaved to the mature large subunit p19 and small subunit p12. Similar results were exhibited in $S$. frugiperda [14]. The $N$-terminal segment of the large subunit of Cscaspase-1 lies near the TETDG motifs, containing the activation cleavage site (Figure 1C). In a word, Cscaspase-1 plays a role of effector in the apoptosis of $C$. suppressalis.

\subsection{Phylogenetic Analysis}

We used CLUSTALX and MEGA 5.0 phylogenetic analysis to compare Cscaspase-1 with other caspases. Figure 2 illustrates the phylogenetic tree constructed by the neighbor joining method. The newly sequenced $C$. suppressalis is most closely related to Eacaspase-1, to which they are $85 \%$ identical at the amino acid level. The Cscaspase-1 shares 62\% identity with DrICE (Drosophila melanogaster) and 38\% identity with human caspase-3. The phylogenetic analysis of insect caspase-1 has revealed the divorce between Lepidoptera and Diptera. Moreover, in Lepidoptera, all months of Noctuidae fell into the well-supported cluster (Figure 2). 
Figure 2. Neighbor-joining phylogenetic tree showing the C. suppressalis (Cscaspase-1, I2EC37) with respect to the other caspases. Heliothis subflexa (Hscaspase-1, G0XQE5), Heliothis virescens (Hvcaspase-1, G0XQE6), Helicoverpa armigera (Hacaspase-1, A9QOJ3), Trichoplusia ni (Tncaspase-1, B6EEC1), Mamestra brassicae (Mbcaspase-1, G0XQE8), Spodoptera exigua (Secaspase-1, G1ELA2), Spodoptera frugiperda (Sfcaspase-1, P89116), Spodoptera littoralis (Slscaspase-1, Q8I955), Spodoptera litura (Slacaspase-1, H6V6K4 ), Euphydryas aurinia (Eacaspase-1, F6K5S3), Danaus plexippus (Dpcaspase-1, G6DDV5), Manduca sexta (Mscaspase-1, F6K5R8), Heliconius melpomene (Hmcaspase-1, C6YXH1), Bombyx mori (Bmcaspase-1, H9JBQ1), Lymantria monacha (Lmcaspase-1, G0XQE7), Plutella xylostella (Pxcaspase-1, D9IVD4), Galleria mellonella (Gmcaspase-1, G0XQE4), Aedes aegypti (Aacaspase-1, Q16MZ1), GJ24096 (Drosophila virilis, B4JM0P7), DrICE (Drosophila melanogaster, O01382), GM12823 (Drosophila sechellia, B4HZF1), GE23886 (Drosophila yakuba, B4PPJ9), Cqcaspase (Culex quinquefasciatus, B0WPG0), Hscaspase-3 (Homo sapiens, P42574). Numbers on the branches are the bootstrap values obtained from 1000 replicates (only bootstrap values $>50$ are shown).

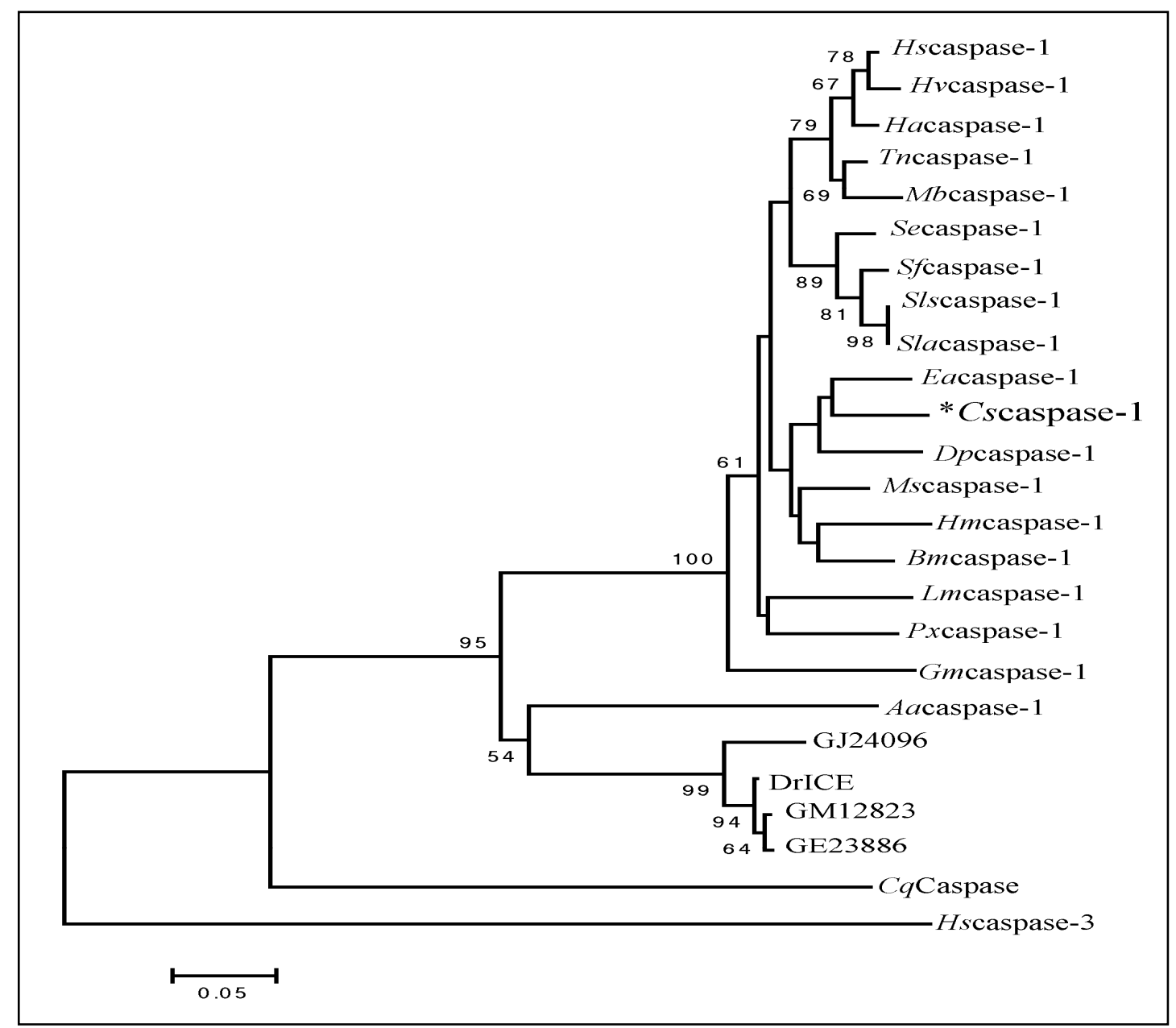

\subsection{Genomic Analysis of Cscaspase-1}

A pair of Cscaspase-1 specific primers was used to amplify a 985 bp DNA fragment from C. suppressalis (Figure 3). Hereafter the PCR products were sequenced. Comparative alignment of the 
genomic sequence with corresponding cDNA sequence showed that there was no intron in the genome of Cscaspase-1. The negative correlation between intron size and gene expression level was suggested in some studies, and the shorter or no introns genes had highly expressed level [21-23]. However, apoptosis is very important to lead to various biological processes, such as development [24], tissue homeostasis, DNA damage response [25] and stress response [5]. So, the Cscaspase-1 didn't have any intron, in order to need to highly express in various biological processes, on the basis of the least costs of transcription.

Figure 3. The PCR products of Cscaspase-1 DNA in the agarose gel electrophoresis (1.0\%). Lane M contains $2 \mathrm{~kb}$ DNA molecular weight, lanes 1 and 2: the PCR product of Cscaspase-1 DNA, respectively.

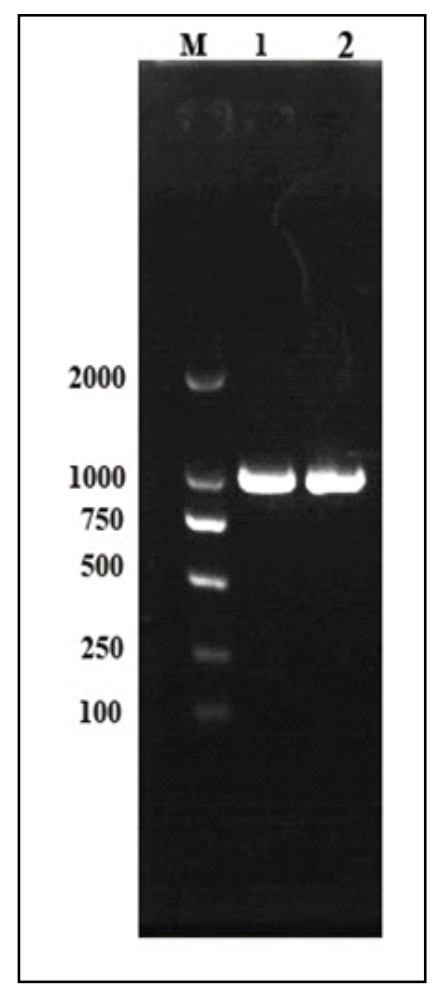

\subsection{Tissues (organs) Distribution of Cscaspase-1mRNA Expression in C. suppressalis Larvae}

The Cscaspase-1 transcripts were found in all tissues of the fifth instar larvae screened in this study, and the higher levels were found in the midgut, hindgut and Malpighian tubules. Interestingly a high expression level was also detected in hemocytes (Figure 4). Similar results were found in Galleria mellonella and Helicoverpa armigera [15,17]. Hindgut and Malpighian tubules reabsorb water, salts, and other substances before excretion in insect. However, some toxic substances, such as uric acid, could induce the apoptosis of cell [26]. The larval midgut epithelium of Lepidoptera degenerates during metamorphosis [27-30]. Since apoptosis plays a central role in tissue remodeling, we would expect to detect higher basal levels of caspases in tissues with rapid renewal rates. The haemolymph in which all the internal organs are bathed might migrate the Cscaspase- 1 to any organ had to execute the apoptosis. 
Figure 4. Relative mRNA expression levels of the Cscaspase-1 gene in different tissues (organs) of the $C$. suppressalis larvae. Abbreviation: HG, hindgut, MG, midgut, FG, foregut, HC, haemocytes, MT, Malpighian tubules, EP, epidermis, FB, fat body, HE, Head. Values are denoted as mean $\pm \mathrm{SE}$. The quantity of each Cscaspase- 1 mRNA is normalized to the abundance of 18SrRNA.

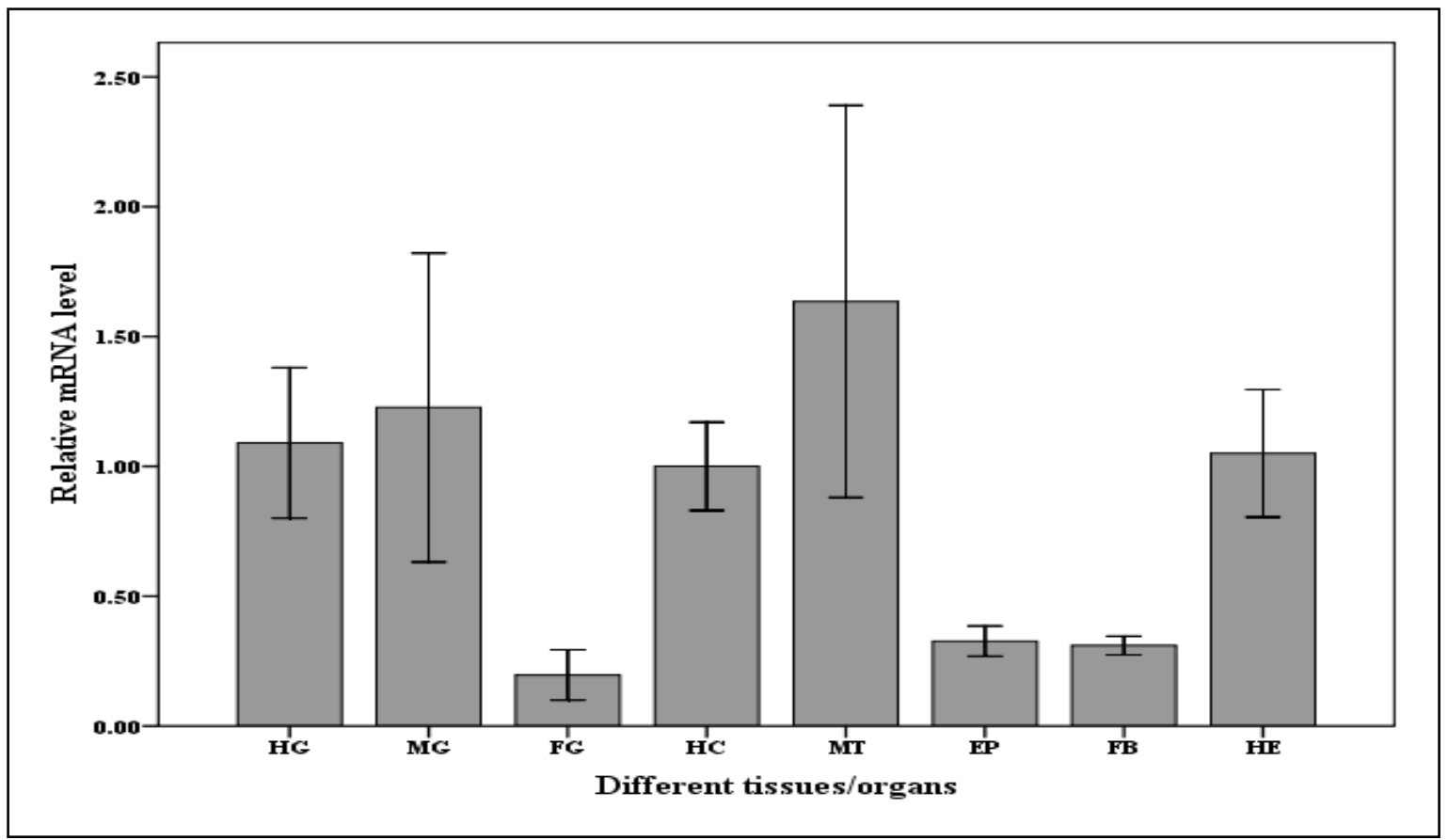

\subsection{Expression of Cscaspase-1 in the Developmental Stages of the C. suppressalis}

In order to determine Cscaspase-1 exhibit developmental expression patterns, we analyzed basal expression in the eggs, first, second, third, fourth, fifth, sixth instar larvae, pupae and adults of C. suppressalis. The results demonstrated unexpectedly the lowest expression level of Cscaspase-1 was observed in the eggs. Then the level was increased by the development of $C$. suppressalis. The most abundance was detected in female pupae. The results also profiled the Cscaspase- 1 mRNA had a higher level in female pupae than those of male (Figure 5). It is obvious when looking at a mature organism that it is the product of a series of cellular production and degradation events. Especially, the development of holometabolous insects is characterized by a complete metamorphosis. Examination of Cscaspase-1 expression in different developmental stages indicated low constitutive levels in the eggs and early larvae stage. In insects, the steroid hormone ecdysone regulates the genes of caspases [11,15,31,32]. The growth and metamorphosis of insects from embryo to adult, including each larval molting, are triggered by pulses of ecdysone. The similar results showed two ecdysone pulses happened respectively at the end of third larval instar and early pupal stage in Drosophila [33-35]. Totally, we concluded that the Cscaspase-1 could contribute to the development of C. suppressalis. 
Figure 5. Relative mRNA expression levels of the Cscaspase-1 gene in different developmental stages of the C. suppressalis. Abbreviation: E, eggs, L1, the first instar larvae, L2, the second instar larvae, L3, the third instar larvae, L4, the fourth instar larvae, L5, the fifth instar larvae, L6, the sixth instar larvae, P, pupae, A, Adults. Values are denoted as mean $\pm \mathrm{SE}$. The quantity of $C$ scaspase- $1 \mathrm{mRNA}$ is normalized to the abundance of 18SrRNA.

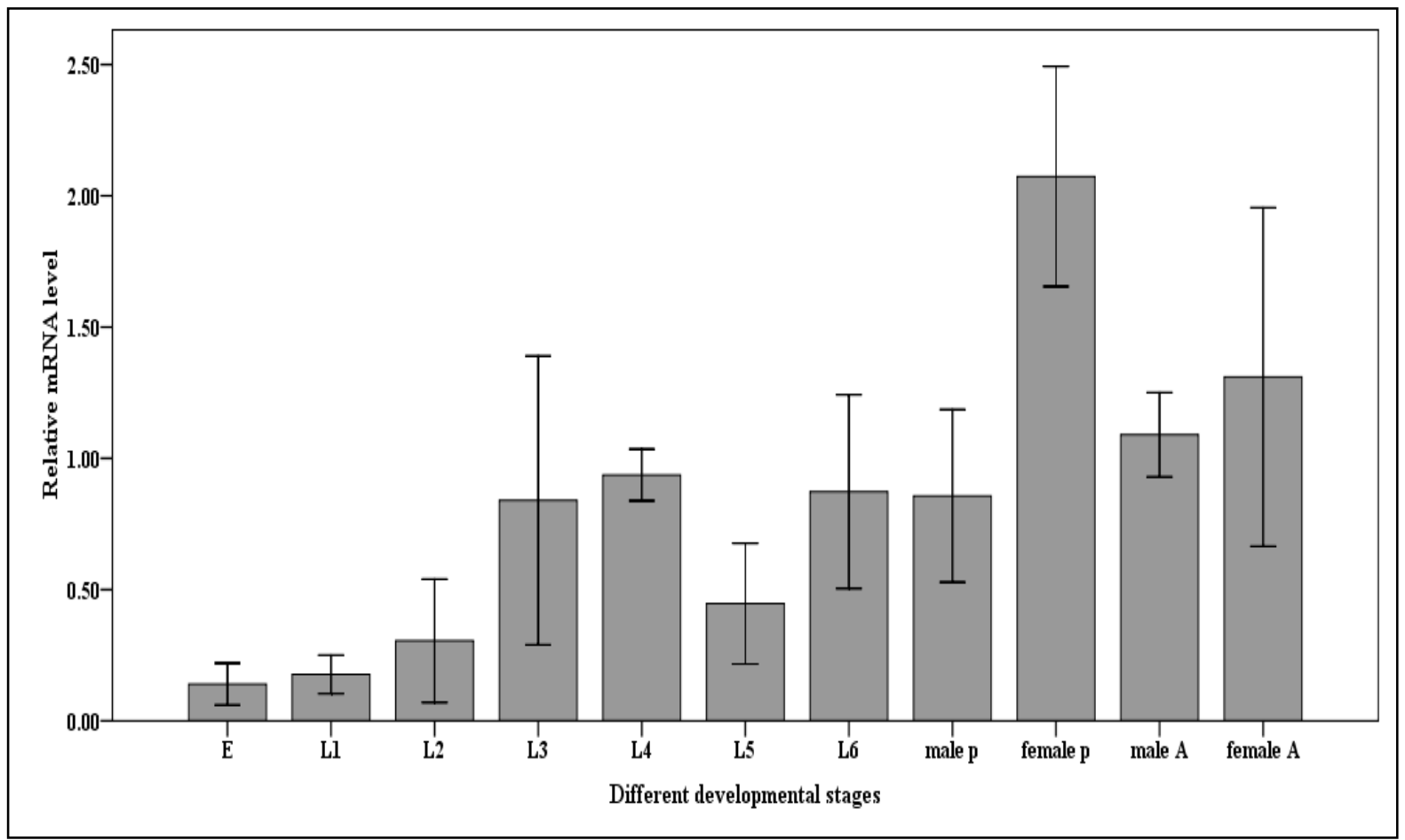

\subsection{Expression of Cscaspase-1 mRNA under Various Temperatures}

In present work, the relative mRNA levels of Cscaspase-1 of $C$. suppressalis were tested at temperature gradients from $-11{ }^{\circ} \mathrm{C}$ to $42{ }^{\circ} \mathrm{C}$. The level was increased in larvae treated at some temperatures, and the highest level that were reached to 6.7 fold as compared to that of control temperature was observed in larvae exposed at $-3{ }^{\circ} \mathrm{C}, 2 \mathrm{~h}$. During heat stress, the Cscaspase- 1 level reached maximum level at $36{ }^{\circ} \mathrm{C}$, which increased 2.9 fold of control. There were different expression trends between high and low temperature in this study. For example, the mRNA level of Cscaspase-1 under low temperature fluctuated strongly comparing to the heat (Figure 6). Our studies showed that the Cscaspase-1 transcript can be upregulated under heat and cold stress. The two highest increases were at $-3{ }^{\circ} \mathrm{C}$ and $36{ }^{\circ} \mathrm{C}$. In $C$. suppressalis, Cscaspase- 1 could be coordinated to encounter with the following temperature stress. When the specimens are exposed to extreme temperature, some cells can produce the apoptosis in order to decrease the cost of survival. 
Figure 6. Relative expression levels of the Cscaspase- 1 in the rice stem borer under temperature stress. The larvae was exposed for $-11{ }^{\circ} \mathrm{C},-8{ }^{\circ} \mathrm{C},-6{ }^{\circ} \mathrm{C},-3{ }^{\circ} \mathrm{C}, 0{ }^{\circ} \mathrm{C}, 3{ }^{\circ} \mathrm{C}$, $6{ }^{\circ} \mathrm{C}, 9{ }^{\circ} \mathrm{C}, 12{ }^{\circ} \mathrm{C}, 15^{\circ} \mathrm{C}, 18^{\circ} \mathrm{C}, 21^{\circ} \mathrm{C}, 24{ }^{\circ} \mathrm{C}, 27^{\circ} \mathrm{C}, 30^{\circ} \mathrm{C}, 33{ }^{\circ} \mathrm{C}, 36{ }^{\circ} \mathrm{C}, 39^{\circ} \mathrm{C}$ and $42{ }^{\circ} \mathrm{C}$ for $2 \mathrm{~h}$. Values are denoted as mean $\pm \mathrm{SE}$. The quantity of each Cscaspase- $1 \mathrm{mRNA}$ is normalized to the abundance of 18 SrRNA.

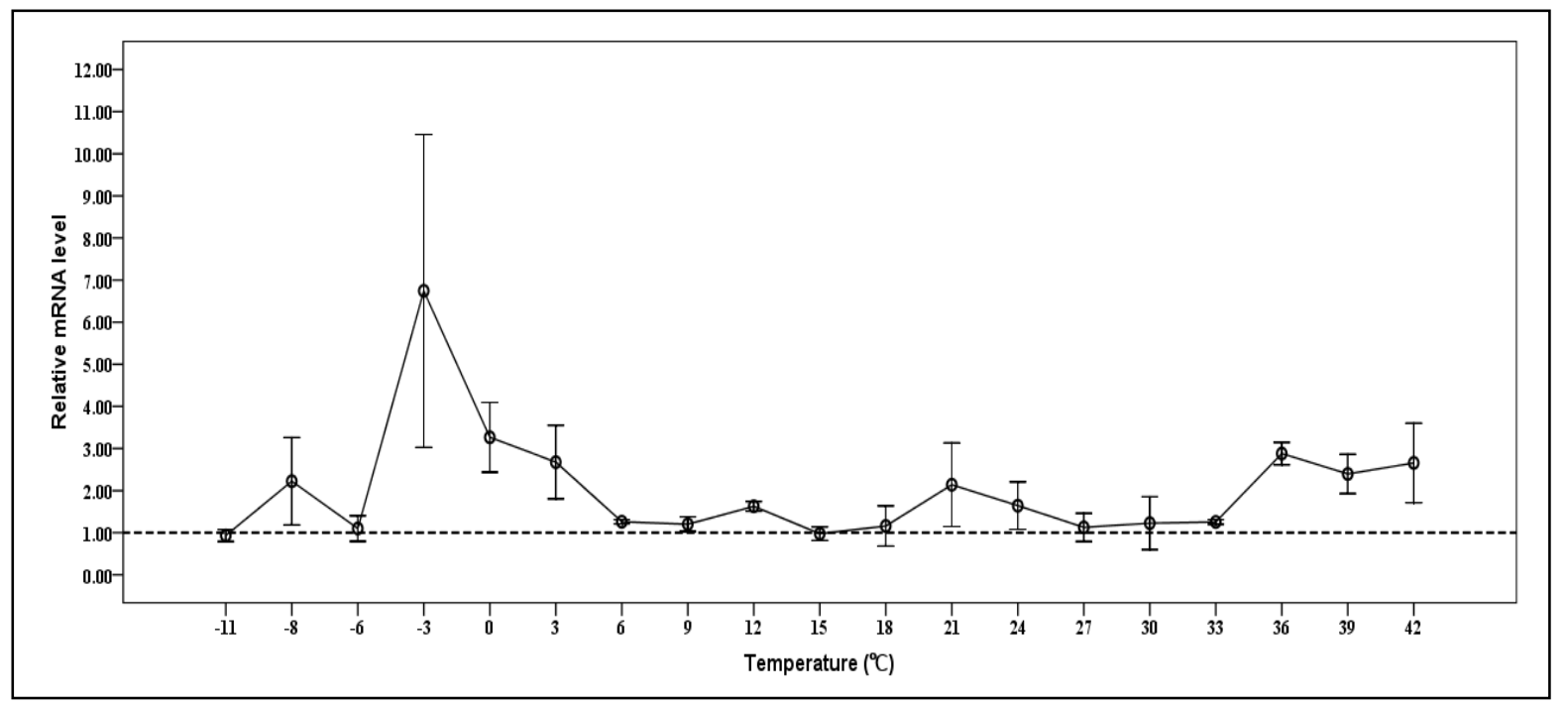

\section{Experimental Section}

\subsection{Insects}

The population of $C$. suppressalis was collected from the suburb of Yangzhou $\left(32.39^{\circ} \mathrm{N}, 119.42^{\circ} \mathrm{E}\right)$. The rice striped borers were reared in an environmental chamber at $28 \pm 1{ }^{\circ} \mathrm{C}, 16: 8$ (L:D) and $\mathrm{RH}=70 \% \pm 5 \%[36]$.

Table 1. Primers used in the cDNA cloning, DNA analysis and real-time quantitative PCR.

\begin{tabular}{cc}
\hline Primers & Sequences $\mathbf{5}^{\prime}-\mathbf{3}$ ') \\
\hline DP-F & AYCAYGARCATTTYGAHATTCACA \\
DP-R & DCCACCATCCRATTTATCACCTTG \\
caspase-1 5' & CCTGTGCGAGATTTCAAGCTGTGA \\
caspase-1 3' & ACTTTACTGCTGACAAGTGCCCTTCC \\
caspase-1-F & TGCATGTCATTTCGGTTGTTG \\
caspase-1-R & CTCCGCCGTTTTCCCTTTG \\
GSP-1 & CGAAATGTCTGACGAAG \\
GSP-2 & AAACTAGGGCAAACTCA \\
18SrRNA-F & CACGGGAAATCTCACCAGG \\
18SrRNA-R & CAGACAAATCGCTCCACCAACTA \\
UPM(Long) & CTAATACGACTCACTATAGGGCAAGCAGTGGTATCAACGCAGAGT \\
UPM(Short) & CTAATACGACTCACTATAGGGC \\
\hline
\end{tabular}

Note: DP, degenerate primers and UPM, universal primer mix.

\subsection{Cloning and RACEs}

Total RNA was extracted by the SV Total RNA isolation system (Promega Z3100) combined with DNase digestion to eliminate DNA contamination. Total cDNA was synthesized by oligo(dT $)_{18}$ primer 
(TaKaRa). The full-length cDNA of the caspase-1 gene was determined using 5'- and 3'-RACE (SMART RACE, Clontech). The primers used are shown in Table 1. To ensure that the 5 ' and $3^{\prime}$ fragments were from the same gene, specific primer were designed, then used to PCR amplify the sequences.

\subsection{Characterization of the Cscaspase-1 Genome}

The genomic DNA of $C$. suppressalis was extracted by Axyprep ${ }^{\mathrm{TM}}$ multisource Genomic DNA Kit (Axygen, USA). A pair of specific primers (GSP-1 and GSP-2) flanking the ORF was designed to amplified Cscaspase-1 genomic fragment (Table 1). A touch-down PCR was used, and the parameters are as follows: $94{ }^{\circ} \mathrm{C}$ for $5 \mathrm{~min}, 15$ cycles at $94{ }^{\circ} \mathrm{C}$ for $30 \mathrm{~s}, 65-50{ }^{\circ} \mathrm{C}$ (decreasing by $-1{ }^{\circ} \mathrm{C} /$ cycle) for $30 \mathrm{~s}$, and $72{ }^{\circ} \mathrm{C}$ for $1 \mathrm{~min} 30 \mathrm{~s}$, followed by 30 cycles of $94{ }^{\circ} \mathrm{C}$ for $30 \mathrm{~s}, 50{ }^{\circ} \mathrm{C}$ for $30 \mathrm{~s}$, and $1 \mathrm{~min} 30 \mathrm{~s}$, and a final extension at $72{ }^{\circ} \mathrm{C}$ for $10 \mathrm{~min}$. Hereafter the PCR amplified fragment was sequenced.

\subsection{Sample Preparation}

The rice striped borers were reared successively to third generation in the seedlings. Then, the egg masses, the first, second, fourth, fifth, sixth instar larvae, pupae (male and female), and one-day adults (male and female), were randomly selected for the experiment. Each experiment was repeated three times. The fifth instar larvae were anesthetized on ice for $15 \mathrm{~min}$ before dissection. Head (HE), epidermis (EP), fat body (FB), foregut (FG), midgut (MG), hindgut (HG), Malpighian tubules (MT), and haemocytes (HC) were collected and rinsed with $0.9 \%$ sodium chloride solution. The samples were frozen immediately in liquid nitrogen and stored at $-80{ }^{\circ} \mathrm{C}$ until the experiment.

\subsection{Temperature Stress}

Larvae used in experiments were all 5 th instars of similar body size and were assigned randomly to each experimental group. Each experimental group contained 10 larvae; each larva was confined individually in glass tubes and exposed to a given temperature (including $-11^{\circ} \mathrm{C},-8{ }^{\circ} \mathrm{C},-6{ }^{\circ} \mathrm{C},-3{ }^{\circ} \mathrm{C}$, $0{ }^{\circ} \mathrm{C}, 3{ }^{\circ} \mathrm{C}, 6{ }^{\circ} \mathrm{C}, 9{ }^{\circ} \mathrm{C}, 12{ }^{\circ} \mathrm{C}, 15{ }^{\circ} \mathrm{C}, 18{ }^{\circ} \mathrm{C}, 21{ }^{\circ} \mathrm{C}, 24{ }^{\circ} \mathrm{C}, 27{ }^{\circ} \mathrm{C}, 30{ }^{\circ} \mathrm{C}, 33{ }^{\circ} \mathrm{C}, 36{ }^{\circ} \mathrm{C}, 39{ }^{\circ} \mathrm{C}$, and $42{ }^{\circ} \mathrm{C}$ ) for $2 \mathrm{~h}$ in a constant temperature subzero incubator (DC-3010, Jiangnan equipment). The larvae were recovered for $2 \mathrm{~h}$. Survived larvae (Table S1) were frozen in liquid nitrogen and then stored at $-70{ }^{\circ} \mathrm{C}$. Each treatment included more than three individual larvae.

\subsection{Quantitative Real-time PCR (qPCR) Analysis}

Total RNA was extracted by the SV Total RNA isolation system (Promega Z3100), followed by DNase treatment to eliminate DNA contamination. The reaction volume was $20 \mu \mathrm{L}$. Each reaction contained $10 \mu \mathrm{L}$ of $2 \times \mathrm{SYBR}^{\circledR}$ Premix EXTaqTM (TaKaRa, Dalian, China) master mix, $0.8 \mu \mathrm{L}$ of each of gene specific primers (caspase-1-F and caspase-1-R) (Table 1), $0.4 \mu \mathrm{L}$ of Rox reference Dye, and $2 \mu \mathrm{L}$ of cDNA templates. The annealing temperature was $62.9^{\circ} \mathrm{C}$. Reactions were carried out on a CFX-96 real-time PCR system (Bio-Rad). The efficiencies of the target and reference genes are similar. The quantity of Cscaspase-1 mRNA was calculated using the $2^{-\Delta \Delta \mathrm{Ct}}$ method [37], and normalized to the abundance of the 18SrRNA gene. Following qPCR, the homogeneity of the PCR products was confirmed by melting curve analysis. 


\subsection{Bioinformatic Analysis}

The open reading frames (ORFs) were identified with the aid of the ORF Finder software [38]. The deduced amino acid sequences were aligned using ClustalX software. Sequence analysis tools of the ExPASy Molecular Biology Server of Swiss Institute of Bioinformatics, including Translate, Compute pI/MW and Blast were used to analyze the deduced caspase-1 protein sequence. Amino acid sequences were used to estimate phylogeny with the neighbor-joining, minimum evolution, maximum likelihood and maximum parsimony methods. Phylogenetic trees were constructed with 1000 bootstrap replicates using MEGA version 5.0 [39].

\subsection{Computational Molecular Modeling}

Homology models were generated using Protein Homology/analogy recognition engine V 2.0 [40]. Briefly, the caspase-1 sequence was aligned by the Phyre2, and the best model of Spodoptera frugiperda X-ray D* structure (PDB ID: 2NN3) was used for modeling analyses. The Chimera Tool was used to visualize the three-dimensional coordinates for the atoms of the model [41].

\section{Conclusions}

In this study, we successfully isolated and characterized Cscaspase-1 of C. suppressalis. Cscaspase-1 provides us with the opportunity for a study on the role of caspase family in development, metamorphosis and the role of apoptosis in regulating thermotolerance of insect. However, regulations of caspases in the apoptosis were a highly complex and dynamic process. Therefore, we are undertaking characterizations and further analysis of other caspases in C. suppressalis, including initiator and effector. Caspase cleavage is required for activation, while induction of mRNA does not completely indicate activation. Thus, we plan further study on the cleavage activation of Cscaspase-1. We expect that investigations of caspases will play key roles in the integrated management of $C$. suppressali in the future.

\section{Acknowledgments}

This work was supported by the National Basic Research and Development Program of China (2012CB114100) and Ph.D. Programs Foundation of Ministry of Education of China (20113250110008).

\section{References}

1. Kerr, J.F.R.; Wyllie, H.A.; Currie, R.A. Apoptosis: A basic biological phenomenon with wide-ranging Implications in Tissue Kinetics. Br. J. Cancer 1972, 26, 239-257.

2. White, E. Life, death, and the pursuit of apoptosis. Gene. Dev. 1996, 10, 1-15.

3. Teodoro, J.G.; Branton, P.E. Regulation of apoptosis by viral gene products. J. Virol. 1997, 71, 1739-1746.

4. Benedict, C.A.; Norris, P.S.; Ware, C.F. To kill or be killed: Viral evasion of apoptosis. Nat. Immunol. 2002, 3, 1013-1018.

5. Yi, S.X.; Moore, C.W.; Lee, R.E., Jr. Rapid cold-hardening protects Drosophila melanogaster from cold-induced apoptosis. Apoptosis 2007, 12, 1183-1193. 
6. Cohen, G.M. Caspases: The executioners of apoptosis. Biochem. J. 1997, 326, 1-16.

7. Thornberry, N.A.; Lazebnik, Y. Caspases: Enemies within. Science 1998, 281, 1312-1316.

8. Raff, M. Cell suicide for beginners. Nature 1998, 396, 119-122.

9. Clem, R.J. The Role of Apoptosis in Defense against Baculovirus Infection in Insects. In Role of Apoptosis in Infection: Current Topics in Microbiology and Immunology; Griffin, D., Ed.; Springer: Heidelberg, Germany, 2005; Volume 289, pp. 113-129.

10. Richardson, H.; Kumar, S. Death to flies: Drosophila as a model system to study programmed cell death. J. Immunol. Methods 2002, 265, 21-38.

11. Cooper, D.M.; Thi, E.P.; Chamberlain, C.M.; Pio, F.; Lowenberger, C. Aedes Dronc: A novel ecdysone-inducible caspase in the yellow fever mosquito, Aedes aegypti. Insect Mol. Biol. 2007, 16, 563-572.

12. Zhang, J.Y.; Pan, M.H.; Sun, Z.Y.; Huang, S.J.; Yu, Z.S.; Liu, D.; Zhao, D.H.; Lu, C. The genomic underpinnings of apoptosis in the silkworm, Bombyx mori. BMC Genomics 2010, 11, 611.

13. Courtiade, J.; Pauchet, Y.; Vogel, H.; Heckel, D.G. A comprehensive characterization of the caspase gene family in insects from the order Lepidoptera. BMC Genomics 2011, 12, 357.

14. Forsyth, C.M.; Lemongello, D.; LaCount, D.J.; Friesen, P.D.; Fisher, A.J. Crystal Structure of an Invertebrate Caspase. J. Biol. Chem. 2004, 279, 7001-7008.

15. Yang, D.T.; Chai, L.Q.; Wang, J.X.; Zhao, X.F. Molecular cloning and characterization of Hearm caspase-1 from Helicoverpa armigera. Mol. Biol. Rep. 2008, 35, 405-412.

16. Zhuang, H.M.; Wang, K.F.; Miyata, T.; Wu, Z.J.; Wu, G.; Xie, L.H. Identification and expression of caspase-1 gene under heat stress in insecticide susceptible and-resistant Plutella xylostella (Lepidoptera: Plutellidae). Mol. Biol. Rep. 2011, 38, 2529-2539.

17. Khoa, D.B.; Trang, L.T.D.; Takeda, M. Expression analyses of caspase-1 and related activities in the midgut of Galleria mellonella during metamorphosis. Insect Mol. Biol. 2012, 21, 247-256.

18. Bowler, K.; Terblanche, J.S. Insect thermal tolerance: What is the role of ontogeny, ageing and senescence? Biol. Rev. 2008, 83, 339-355.

19. Doeppner, T.R.; Grune, T.; de Groot, H.; Rauen, U. Cold induced apoptosis of rat liver endothelial cells: Involvement of the proteasome. Transplantation 2003, 75, 1946-1953.

20. Lu, M.X.; Liu, Z.X.; Wang, X.; Du, Y.Z. Seasonal Cold Tolerance of Chilo suppressalis (Walker) in Yangzhou, China. Ann. Entomol. Soc. Am. 2012, 105, 479-483.

21. Hurst, L.D.; McVean, G.; Moore, T. Imprinted genes have few and small introns. Nat. Genet. 1996, 12, 234-237.

22. Castillo-Davis, C.I.; Mekhedov, S.L.; Hartl, D.L.; Koonin, E.V.; Kondrashov, F.A. Selection for short introns in highly expressed genes. Nat. Genet. 2002, 31, 415-418.

23. Comeron, J.M. Selective and mutational patterns associated with gene expression in humans: Influences on synonymous composition and intron presence. Genetics 2004, 167, 1293-1304.

24. Penaloza, C.; Orlanski, S.; Ye, Y.; Entezari-Zaher, T.; Javdan, M.; Zakeri, Z. Cell death in mammalian development. Curr. Pharm. Des. 2008, 14, 184-196.

25. Amundson, S.A.; Myers, T.G.; Fornace, A.J. Roles for p53 in growth arrest and apoptosis: Putting on the brakes after genotoxic stress. Oncogene 1998, 17, 3287-3299.

26. McGavin, G.C. Essential Entomology: An Order by Order Introduction; Oxford University Press: New York, NY, USA, 2001. 
27. Uwo, M.F.; Ui-Tei, K.; Park, P.; Takeda, M. Replacement of midgut epithelium in the greater wax moth, Galleria mellonela, during larval-pupal moult. Cell Tissue Res. 2002, 308, 319-331.

28. Tettamanti, G.; Grimaldi, A.; Casartelli, M.; Ambrosetti, E.; Ponti, B.; Congiu, T.; Ferrarese, R.; Rivas-Pena, M.L.; Pennacchio, F.; Eguileor, M. Programmed cell death and stem cell differentiation are responsible for midgut replacement in Heliothis virescens during prepupal instar. Cell Tissue Res. 2007, 330, 345-359.

29. Vilaplana, L.; Pascual, N.; Perera, N.; Belles, X. Molecular characterization of an inhibitor of apoptosis in the Egyptian armyworm, Spodoptera littoralis, and midgut cell death during metamorphosis. Insect Biochem. Mol. Biol. 2007, 37, 1241-1248.

30. Hakim, R.S.; Baldwin, K.; Smagghe, G. Regulation of midgut growth, development, and metamorphosis. Annu. Rev. Entomol. 2010, 55, 593-608.

31. Kilpatrick, Z.E.; Cakouros, D.; Kumar, S. Ecdysone-mediated up-regulation of the effector caspase DRICE is required for hormone-dependent apoptosis in Drosophila cells. J. Biol. Chem. 2005, 280, 981-986.

32. Yin, V.P.; Thummel, C.S. Mechanisms of steroid-triggered programmed cell death in Drosophila. Semin. Cell Dev. Biol. 2005, 16, 237-243.

33. Baehrecke, E.H. Steroid regulation of programmed cell death during Drosophila development. Cell Death Differ. 2000, 7, 1057-1062.

34. Jiang, C.; Jiang, C.; Lamblin, A.F.; Steller, H.; Thummel, C.S. A steroid-triggered transcriptional hierarchy controls salivary gland cell death during Drosophila metamorphosis. Mol. Cell 2000, 5, $445-455$.

35. Lee, C.Y.; Cooksey, B.; Baehrecke, E.H. Steroid regulation of midgut cell death during Drosophila development. Dev. Biol. 2002, 250, 101-111.

36. Shang, Z.Z.; Wang, Y.S.; Zou, Y.H. Study on rearing method of rice stem borer Chilo suppressalis Walker (in Chinese). Acta Entomol. Sin. 1979, 2, 164-167.

37. Schmittgen, T.D.; Livak, K.J. Analyzing real-time PCR data by the comparative CT method. Nat. Protoc. 2008, 3, 1101-1108.

38. ORF Finder. Available online: http://www.ncbi.nlm.nih.gov/gorf/gorf.html (accessed on 13 May 2013).

39. Tamura, K.; Peterson, D.; Peterson, N.; Stecher, G.; Nei, M.; Kumar, S. MEGA5: Molecular Evolutionary Genetics Analysis using Maximum Likelihood, Evolutionary Distance, and Maximum Parsimony Methods. Mol. Biol. Evol. 2011, 28, 2731-2739.

40. Kelley, L.A.; Sternberg, M.J.E. Protein structure prediction on the web: A case study using the phyre server. Nat. Protoc. 2009, 4, 363-371.

41. Pettersen, E.F.; Goddard, T.D.; Huang, C.C.; Couch, G.S.; Greenblatt, D.M.; Meng, E.C.; Ferrin, T.E. UCSF Chimera-a visualization system for exploratory research and analysis. J. Comput. Chem. 2004, 25, 1605-1612.

(C) 2013 by the authors; licensee MDPI, Basel, Switzerland. This article is an open access article distributed under the terms and conditions of the Creative Commons Attribution license (http://creativecommons.org/licenses/by/3.0/). 